\title{
NUMERICAL SIMULATION OF THE TRANSIENT FLOW BEHAVIOUR IN TUBE BUNDLES USING A VOLUME PENALISATION METHOD
}

\author{
Kai Schneider \\ Laboratoire de Modélisation et Simulation Numérique en Mécanique, \\ CNRS and Universités d'Aix-Marseille \\ \& Centre de Mathématiques et d'Informatique, Université de Provence, \\ 39 rue F. Joliot-Curie, 13453 Marseille - Cedex 13, France \\ Marie Farge \\ Laboratoire de Météorologie Dynamique, CNRS, \\ Ecole Normale Supérieure, \\ 24 rue Lhomond, 75231 Paris - Cedex 05, France
}

\begin{abstract}
We present high resolution numerical simulations of incompressible two-dimensional flows in tube bundles, staggered or inline, as encountered in heat exchangers or chemical reactors. We study the time evolution of several flows in arrays of cylinders or squares, at Reynolds number 200. The numerical scheme is either based on adaptive wavelet or Fourier pseudo-spectral space discretization with adaptive time stepping. A volume penalisation method is used to impose no-slip boundary conditions on the tubes. Lift and drag coefficients for the different geometries of tube bundles are compared and perspectives for fluid-structure interaction are given.
\end{abstract}

\section{INTRODUCTION}

The numerical simulation of turbulent flows in complex geometries is a key challenge in computational fluid dynamics (CFD). Grid generation and turbulence modelling near the wall are crucial, especially to predict lift and drag and to control of the flow. A suitable approach is the volume penalisation method which has been introduced by Arquis and Caltagirone (1984) to compute flows in porous media, and generalized by Angot et al. (1999) to flows past obstacles. In the latter walls or solid obstacles are modelled as porous media whose porosity tends to zero. They also give a mathematical proof of the convergence towards the solution of Navier-Stokes equations with no-slip boundary conditions. In the volume penalisation method the Navier-Stokes equations are modified by adding a Darcy force. Fluid regions are thus considered as fully permeable, and solid regions as almost impermeable. The geometry of the flow is taken into account using a spatially varying permability, which enables an easy implementation and fur- thermore allows obstacles to move, as it is the case for flow induced vibrations. The penalisation method has been applied using finite difference/volume schemes (Angot et al., 1999; Khadra et al., 2000), pseudospectral methods (Forestier et al., 2000; Kevlahan and Ghidaglia, 2001; Schneider, 2004) and recently adaptive wavelet methods (Schneider and Farge, 2002; Farge and Schneider, 2001). The latter scheme automatically adapts the spatial grid not only to the evolution of the flow, but also to the geometry of walls or bluff bodies (Schneider and Farge, 2002).

The paper is organized as follows: first we present the penalisation method together with the numerical schemes, i.e. Fourier and wavelet, used to solve the penalized Navier-Stokes equations. We then study several applications to tube bundles, as encountered in heat exchangers, static mixers and chemical reactors. We consider both cylindrical and square cross sections, for either inline or staggered arrangements. We compute the drag and lift coefficients for different geometries, and discuss the influence of the angle of attack of the flow. Finally, we give some conclusions and perspectives for three-dimensional flows.

\section{VOLUME PENALISATION AND NUMERICAL DISCRETISATION}

\subsection{Penalized equations}

The volume penalisation method has been proposed by Arquis and Caltagirone (1984). Its is based on the physical idea which consists in modelling solid walls or obstacles as porous media whose porosity tends to zero. The geometry of the flow is described by a mask function $\chi(\vec{x})$ which is 1 inside the solid regions and 0 elsewhere. Obstacles which move or with timevarying shape can be taken into account by simply using a time-dependent mask function. It is hence 
particularly suited to compute fluid-structure interaction. The incompressible Navier-Stokes equations are modified by adding a forcing term containing the mask function:

$$
\begin{aligned}
\partial_{t} \vec{u}_{\eta}+\vec{u}_{\eta} & \cdot \nabla \vec{u}_{\eta}+\nabla p_{\eta}-\nu \nabla^{2} \vec{u}_{\eta} \\
= & -\frac{1}{\eta} \chi \Omega_{s}\left(\vec{u}_{\eta}-\vec{u}_{p}(t)\right) \\
& \nabla \cdot \vec{u}_{\eta}=0
\end{aligned}
$$

where $\vec{u}_{\eta}(\vec{x}, t)$ is the flow velocity, $p_{\eta}(\vec{x}, t)$ the pressure, $\vec{u}_{p}(t)$ the obstacle's velocity, $\nu$ the kinematic viscosity, $\eta$ the porosity which tends to zero and where the density is normalized to 1 . The above equations are completed with a suitable initial condition and periodic boundary conditions. The mask function is

$$
\chi_{\Omega_{s}}(\vec{x})= \begin{cases}1 & \text { for } \vec{x} \in \bar{\Omega}_{s} \\ 0 & \text { elsewhere }\end{cases}
$$

where $\bar{\Omega}_{s}$ denotes the set of solid obstacles including their boundaries. For $\eta \longrightarrow 0$ the flow evolution is governed by the Navier-Stokes equation in the fluid regions, and by Darcy's law, i.e. velocity is proportional to pressure gradient, in solid regions. Angot et al. (1999) have proven that the above equations converge towards the Navier-Stokes equations with noslip boundary conditions, the order of convergence being $\eta^{3 / 4}$ inside the obstacle and $\eta^{1 / 4}$ elsewhere. In numerical simulations one finds a convergence of order $\eta$, as reported in (Angot et al., 1999), (Kevlahan and Ghidaglia, 2001).

The hydrodynamic forces $\vec{F}$ exerted by the flow on the obstacle, i.e. drag and lift, are simply computed by integrating the penalized velocity over the obstacle's volume (Angot et al., 1999):

$$
\begin{gathered}
\vec{F}=\lim _{\eta \rightarrow 0} \int_{\Omega_{s}} \nabla p_{\eta} d x=-\lim _{\eta \rightarrow 0} \frac{1}{\eta} \int_{\Omega_{s}} \vec{u}_{\eta} d x \\
=\int_{\partial \Omega_{s}} \sigma(\vec{u}, p) \cdot \vec{n}_{f} d \gamma
\end{gathered}
$$

where $\Omega_{s}$ is the obstacle's volume, $\partial \Omega_{s}$ its boundary, $\vec{n}$ its outer normal and $\sigma(\vec{u}, p)=\frac{1}{2 \nu}\left(\nabla \vec{u}+(\nabla \vec{u})^{t}\right)-$ $p I$ the stress tensor. Hence, the lift and drag forces on the obstacle, i.e. forces parallel and perpendicular to the free-stream velocity of the flow, are easy to compute as volume integrals instead of contour integrals.

For two-dimensional flows the vorticity-velocity formulation is prefered, therefore we take the curl of eq. (1) and get

$$
\begin{array}{r}
\partial_{t} \omega_{\eta}+\left(\vec{u}_{\eta}+\vec{U}_{\infty}\right) \cdot \nabla \omega_{\eta}-\nu \nabla^{2} \omega_{\eta} \\
+\nabla \times\left(\frac{1}{\eta} \chi_{\Omega_{s}}\left(\vec{u}_{\eta}\right)-\vec{u}_{p}(t)\right)=0
\end{array}
$$

where $\omega=\nabla \times \vec{u}$ denotes vorticity and $\vec{U}_{\infty}=$ $\lim _{|\vec{x}| \rightarrow \infty} \vec{u}(\vec{x})$ free-stream velocity.

\subsubsection{Fluid-structure interaction}

Following Khalak and Williamson (1999) the fluidstructure interaction can be modelled by an ordinary differential equation for the position of the obstacle's center of mass $\vec{x}_{p}$

$$
m \frac{d^{2} \vec{x}_{p}}{d t^{2}}+C \frac{d \vec{x}_{p}}{d t}+K \vec{x}_{p}=\vec{F}
$$

where $m$ is the obstacle's mass, $C$ the mechanical damping and $K$ the spring constant. Note that $\vec{F}$ is the fluid force (3) and $\frac{d \vec{x}_{p}}{d t}=\vec{u}_{p}$ the velocity of the obstacle.

\subsection{Numerical discretization}

To solve the penalized Navier-Stokes equations we employ, either a classical Fourier pseudospectral method (Canuto et al., 1988; Kevlahan and Ghidaglia, 2001; Schneider, 2004), or an adaptive wavelet scheme (Fröhlich and Schneider, 1997; Schneider and Farge, 2002). We now describe both methods.

\subsubsection{Fourier pseudo-spectral method}

Fourier pseudo-spectral discretizations are classical schemes in CFD, which are highly accurate for flows with periodic boundary conditions (Canuto et al., 1988). Equation (5) is transformed into Fourier space in order to compute the spatial derivatives and to evolve the vorticity field in time. Terms containing products, i.e. the convection and penalisation terms, are calculated by collocation in physical space. The vorticity field and the velocity are represented as Fourier series

$$
\omega(\vec{x}, t)=\sum_{\vec{k} \in \mathbb{Z}^{2}} \widehat{\omega}(\vec{k}, t) \exp (i \vec{k} \cdot \vec{x})
$$

where the Fourier transform of $\omega$ is defined as

$$
\widehat{\omega}(\vec{k}, t)=\frac{1}{4 \pi^{2}} \int \omega(\vec{x}, t) \exp (-i \vec{k} \cdot \vec{x}) d \vec{x}
$$

with the wavevector $\vec{k}=\left(k_{x}, k_{y}\right)$. The Fourier discretization is uniform in space and eq. (6) is truncated at $k_{x}=-N_{x} / 2$ and $k_{x}=N_{x} / 2+1, k_{y}=-N_{y} / 2$ and $k_{y}=N_{y} / 2+1$, where $N_{x}$ and $N_{y}$ are the number of grid points in $x$ and $y$ direction, respectively. The gradient of $\omega$ is computed by multiplying $\widehat{\omega}$ with 
$i \vec{k}$, the Laplacian by multiplying with $|\vec{k}|^{2}$. The velocity $\vec{u}$ induced by the vorticity $\omega$ is reconstructed in Fourier space using Biot-Savart's law

$$
\vec{u}(\vec{x}, t)=\sum_{\vec{k} \in \mathbb{Z}^{2}, \vec{k} \neq 0} \frac{i \vec{k}^{\perp}}{|\vec{k}|^{2}} \widehat{\omega}(\vec{k}, t) \exp (i \vec{k} \cdot \vec{x})
$$

where $\vec{k}^{\perp}=\left(-k_{y}, k_{x}\right)$.

The convection term $\vec{u} \cdot \nabla \omega$ and the penalisation term $\nabla \times\left(\frac{1}{\eta} \chi_{\Omega_{s}}\left(\vec{u}-\vec{u}_{p}(t)\right)\right.$ are evaluated by the pseudospectral technique using collocation in physical space. To avoid aliasing errors, i.e. the production of small scales due to the nonlinear terms which are not resolved on the grid, we de-aliase at each time step by truncating the Fourier coefficients using the $2 / 3$ rule, i.e.

$$
\widehat{\omega}(\vec{k})=\left\{\begin{array}{lll}
\widehat{\omega}(\vec{k}) & \text { for } & \left(\frac{3 k_{x}}{2 N_{x}}\right)^{2}+\left(\frac{3 k_{y}}{2 N_{y}}\right)^{2}<1 \\
0 & \text { for } & \left(\frac{3 k_{x}}{2 N_{x}}\right)^{2}+\left(\frac{3 k_{y}}{2 N_{y}}\right)^{2} \geq 1
\end{array}\right.
$$

We use Temperton's Fast Fourier Transform which has a complexity of order $N \log _{2} N$, with $N=$ $N_{x} N_{y}$.

For the time discretization we use a semi-implicit scheme with adaptive time-stepping as proposed by Schneider (2004). The linear diffusion term is discretized implicitly using exact time integration, since the Laplace operator is diagonal in Fourier space, and hence no linear system has to be solved. The remaining terms are discretized explicitly using second order Adams-Bashforth extrapolation. This avoids the solution of nonlinear equations, nevertheless it implies a CFL condition, i.e. the maximum size of the time step is limited for stability reasons. However the semi-implicit discretization proposed here improves the stability limit with respect to purely explicit schemes.

For the time step control we compute in each time step $n$ the maximal rms velocity at each grid point,

$$
u_{\max }=\max _{\vec{x}} \quad \sqrt{(u(\vec{x}))^{2}+(v(\vec{x}))^{2}}
$$

and the new time step is

$$
\Delta t_{n+1}=C \Delta x / u_{\max }
$$

with the spatial grid size $\Delta x=\min \left(\frac{L_{x}}{N_{x}}, \frac{L_{y}}{N_{y}}\right)$, where $L_{x}, L_{y}$ denote the length of the domain in $x$ and $y$ directions, respectively, and $C<1$ is the CFL constant.

\subsubsection{Adaptive wavelet method}

As adaptive schemes dynamically adapt the spatial grid at each time step, we first discretize equation (5) in time using semi-implicit finite differences, i.e. Euler-backwards for the viscous term and AdamsBashforth extrapolation for the nonlinear term, which are both second order.

The resulting elliptic problem to be solved at each time step is:

$$
\begin{aligned}
& \left(\gamma I-\nu \nabla^{2}\right) \omega^{n+1}=\frac{4}{3} \gamma \omega^{n}-\frac{1}{3} \gamma \omega^{n-1} \\
& -\nabla \cdot\left(\omega^{\star} \vec{u}^{\star}\right)-\nabla \times\left(\frac{1}{\eta} \chi\left(\vec{u}^{\star}-\vec{u}_{p}\right)\right)
\end{aligned}
$$

where $\omega^{\star}=2 \omega^{n}-\omega^{n-1}$ and $\vec{u}^{\star}=2 \vec{u}^{n}-\vec{u}^{n-1}$, with $\gamma=3 /(2 \Delta t)$ and $I$ the identity.

For the space discretization we use a PetrovGalerkin scheme. The vorticity is developed into a set of trial functions and, to minimize the weighted residual of (10), one requires that the projection onto a space of test functions vanishes. As space of trial functions we employ a two-dimensional periodic multiresolution analysis (MRA) (Farge, 1992) and develop $\omega^{n}$ at time step $n$ into an orthonormal wavelet series

$$
\omega^{n}(x, y)=\sum_{\lambda}\left\langle\omega^{n}, \psi_{\lambda}\right\rangle \psi_{\lambda}(x, y)
$$

with the multi-index $\lambda=\left(j, i_{x}, i_{y}, \mu\right)$, where $j=$ $0, J_{\max }-1$ denotes the scale $2^{-j+1},\left(i_{x}, i_{y}\right)=$ $0, \ldots, 2^{j}-1$ the position and $\mu=1,2,3$ the three directions of the two-dimensional wavelets.

The test functions $\theta_{\lambda}$ are defined as solutions of the linear part of eq. (10)

$$
\left(\gamma I-\nu \nabla^{2}\right) \theta_{\lambda}=\psi_{\lambda}
$$

which are computed in a preprocessing step for each scale only. This avoids assembling the stiffness matrix and solving a linear equation at each time step. The functions $\theta$, called vaguelettes, are explicitely calculated in Fourier space and have similar localization properties as wavelets (Fröhlich and Schneider, 1997). The solution of (10) in wavelet space therewith reduces to a change of basis:

$$
\begin{aligned}
\tilde{\omega}_{\lambda} & =\left\langle\omega^{n+1}, \psi_{\lambda}\right\rangle \\
& =\left\langle\left(\frac{4}{3} \gamma \omega^{n}-\frac{1}{3} \gamma \omega^{n-1}-\nabla \cdot\left(\omega^{\star}\left(\vec{u}^{\star}\right)\right)\right.\right. \\
& \left.\left.-\nabla \times\left(\frac{1}{\eta} \chi\left(\vec{u}^{\star}-\vec{u}_{p}\right)\right)\right), \theta_{\lambda}\right\rangle
\end{aligned}
$$

A nonlinear wavelet thresholding is applied in each time step to obtain an adaptive discretization. One 
only retains only the wavelet coefficients $\tilde{\omega}_{\lambda}$ whose absolute value is above a given threshold $\epsilon=\epsilon_{0} \sqrt{Z}$, where $\epsilon_{0}$ is a constant and $Z=\frac{1}{2} \int|\omega(\vec{x})|^{2} d \vec{x}$ is the total enstrophy. For the next time step the index coefficient set (which addresses each coefficient in wavelet space) is determined by adding neighbours to the retained wavelet coefficients. Consequently, only those coefficients $\tilde{\omega}$ in (13) belonging to this extrapolated index set are computed using the adaptive vaguelette decomposition (Fröhlich and Schneider, 1997). The nonlinear term $-\nabla \cdot\left(\omega^{\star}\left(\vec{u}^{\star}\right)\right)-\nabla \times$ $\left(\frac{1}{\eta} \chi\left(\vec{u}^{\star}-\vec{u}_{p}\right)\right)$ is evaluated by partial collocation on a locally refined grid (Schneider and Farge, 2002), as illustrated in Fig. 1. We find that only 10908 out of 65536 wavelet coefficients, i.e. $16.6 \%$, are used for the computation. The vorticity $\omega^{\star}$ is reconstructed
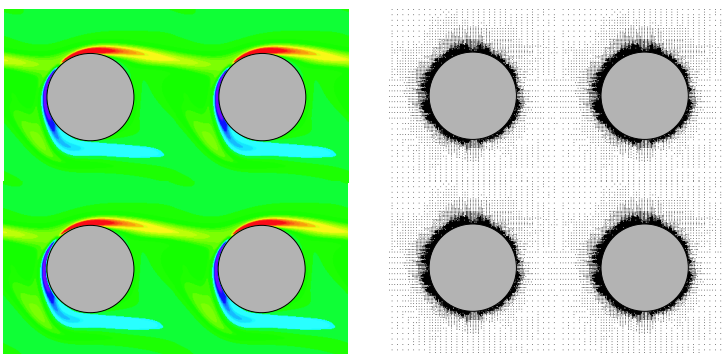

Figure 1: Adaptive wavelet computation of a flow past a tube bundle with circular cross section at $R e=$ 200 for $\alpha=30^{\circ}$. Left: instantaneous vorticity at $t=5$ with maximal resolution $N_{x}=N_{y}=256$ and $\eta=10^{-3}$. Right: corresponding adaptive grid.

in physical space on an adaptive grid from its wavelet coefficients $\tilde{\omega}^{\star}$ using the adaptive wavelet reconstruction algorithm (Fröhlich and Schneider, 1997). From the adaptive vaguelette decomposition with $\theta=$ $\left(\nabla^{2}\right)^{-1} \psi$, we solve $\nabla^{2} \Psi^{\star}=\omega^{\star}$ to get the stream function $\tilde{\Psi}^{\star}$ and reconstruct $\Psi^{\star}$ on a locally refined grid. By means of centered finite differences of 4th order we compute $\nabla \omega^{\star}, \vec{u}^{\star}=\left(-\partial_{y} \Psi^{\star}, \partial_{x} \Psi^{\star}\right)$ and $\nabla \times\left(\frac{1}{\eta} \chi\left(\vec{u}^{\star}-\vec{u}_{p}\right)\right)$ on the adaptive grid. Subsequently, the nonlinear term is summed up pointwise, and finally (13) is solved using the adaptive vaguelette decomposition.

\section{NUMERICAL RESULTS}

In this section we compute the transient flow behaviour in tube bundles at $R e=200$. We consider either circular cross sections or square cross sections and different angles of attack, i.e. $\alpha=0^{\circ}$, corresponding to inline and $\alpha=30^{\circ}, 45^{\circ}$ corresponding to staggered bundles. These configurations are frequently used for cross-flow heat exchangers and static mixers. For the different cases we show flow visualizations of instantaneous vorticity fields $\omega$ and we plot the time evolution of lift and drag coefficients.

\subsection{Tubes with circular cross section}

In Fig. 2 we sketch the flow configuration for bundles of tubes with a circular cross section where $\alpha$ is the angle of attack of the free-stream velocity $\vec{V}_{\infty}$. The geometry is characterized by the pitch to diameter ratio $P / D$, where $D$ denotes the tube's diameter and $P$ the bundle's pitch. In industrial applications the ratio is typically in the range between 1.3 and 2 . We define a Reynolds number based on the tube's diameter and the free-stream velocity, i.e. $R e=V_{\infty} D / \nu$.

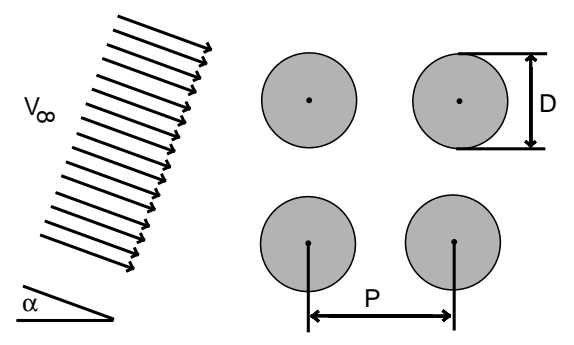

Figure 2: Sketch of the flow configuration: tube bundles with tube pitch $P$, tube diameter $D$ and pitch to diameter ratio $P / D$ (which is typically between 1.3 to 2 ).

In the present simulations we take $D=1, P=2$, and $\alpha=0^{\circ}, 30^{\circ}$ and $45^{\circ}$, corresponding to an in-line, or two staggered arrangements, respectively. The free-stream velocity $\vec{V}_{\infty}$ is normalized to one and the time unit is $\tau=P / V_{\infty}$. The spatial resolution is $N_{x}=N_{y}=256$ which guarantees at least 4 grid points within the boundary layer. The boundary layer thicknessas $\delta$ is evaluated using Prandtl's wall law, i.e. $\delta \propto 1 / \sqrt{R e}$.

In Fig. 3 we show snapshots of the vorticity field at $t=10 \tau$ for three different angles on attack which exhibit different flow behaviours. The inline configuration, i.e. $\alpha=0^{\circ}$, (Fig. 3, top, left), presents four horizontal shear layers which are stable. After a short transition phase, the flow becomes stationary as reflected by a constant drag coefficient (Fig. 4, left). In this case we observe that the flow remains symmetric which is consistent with the vanishing lift coefficient. For the staggered configurations $\alpha=30^{\circ}$ and $45^{\circ}$ we see much stronger production of vorticity at the tubes. A shear layer is thus formed, which becomes unstable, and rolls up into vortices which are periodically shed. This is confirmed by the time evolution of lift and drag coefficients which oscillate with a period of $2 \tau$ (Fig. 4). 

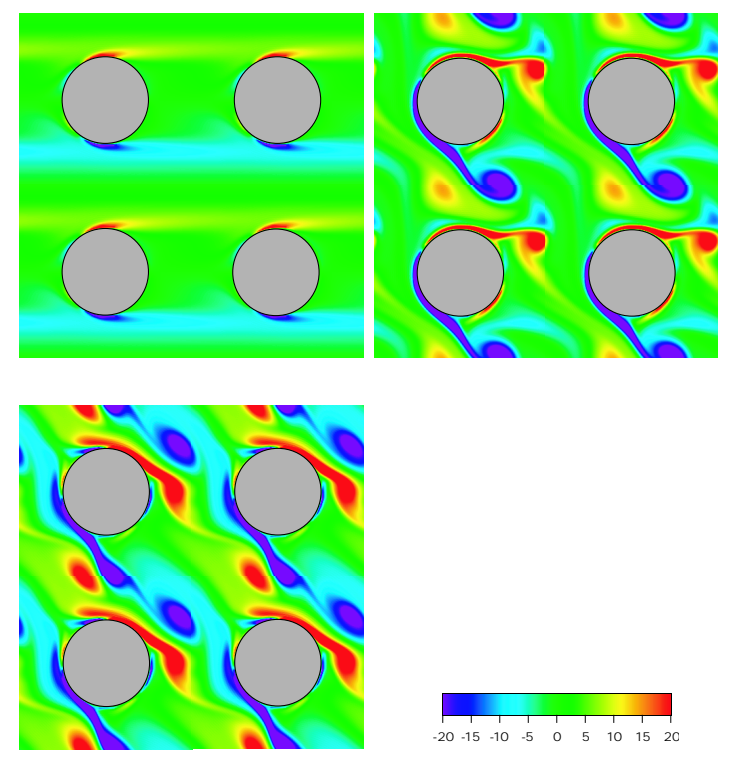

Figure 3: Flow past tube bundles with circular cross section at $R e=200$ with $N_{x}=N_{y}=256$ and $\eta=10^{-3}$. Vorticity fields at $t=10 \tau$ for three angles of incidence, $\alpha=0^{\circ}, 30^{\circ}, 45^{\circ}$.
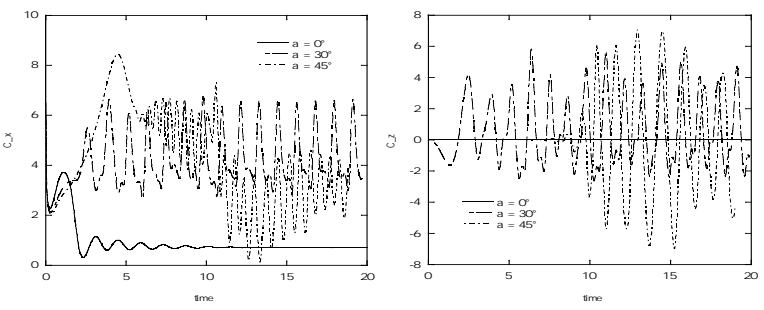

Figure 4: Flow past tube bundles with circular cross section at $R e=200$ for $\alpha=0^{\circ}, 30^{\circ}, 45^{\circ}$. Time evolution of drag (left) and lift (right) coefficients.

\subsection{Tubes with square cross section}

We now consider a bundle of tubes with square cross section at $R e=200$ for three angles of incidence $\alpha=0^{\circ}, 30^{\circ}, 45^{\circ}$. The main difference with the previous case is that here the detachement point of the boundary layer is always located at the corner of the tubes, while for circular tubes its location varies with the Reynolds number and the angle of incidence. We also observe that the vorticity production is increased by a factor of 4 with respect to the circular case.

For the inline geometry $\left(\alpha=0^{\circ}\right)$ we find in both cases a similar behaviour, again four parallel shear layers are being formed (Fig. 5, top, left), and the flow becomes stationary after a transition phase up to $t=5 \tau$. Hence the drag forces are constant and the lift coefficient vanishes (Fig. 6). The flows in the staggered geometries $\left(\alpha=30^{\circ}, 45^{\circ}\right)$ exhibit, like for the circular tubes, the formation of vortices which are however less pronounced (Fig. 5, top, right and bottom, left). For $\alpha=45^{\circ}$ we also see the striking symmetry of the flow, which is broken at later times, around $t=6$ (cf. Fig. 6). In both cases, we observe time oscillations of the drag coefficients, with period $1 \tau$. For $\alpha=30^{\circ}$ we also see a superposition of secondary oscillations with period $0.5 \tau$. We also notice an increase of drag and lift forces by a factor three compared to the circular tubes.
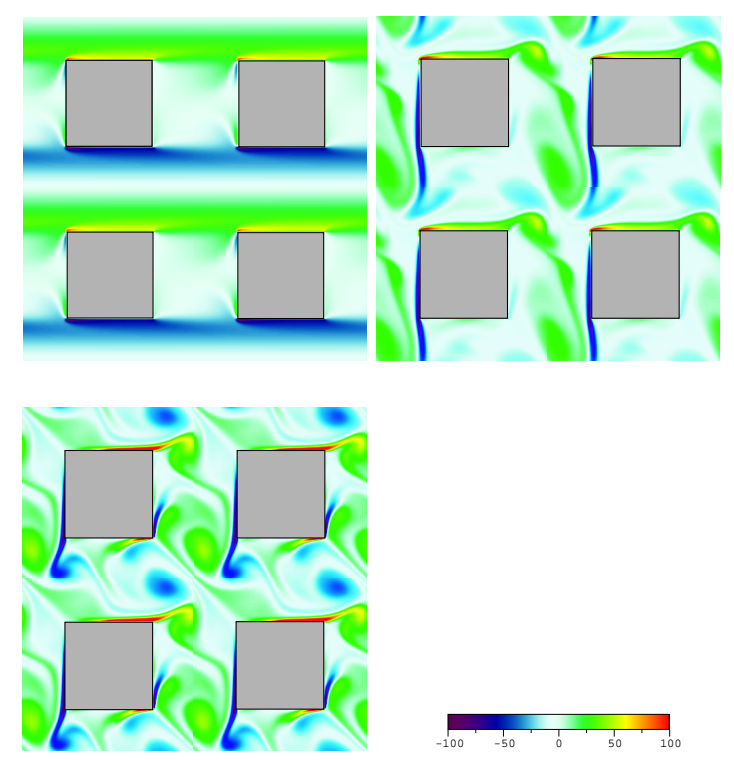

Figure 5: Flow past tube bundles with square cross section at $R e=200$ with $N_{x}=N_{y}=256$ and $\eta=10^{-3}$. Vorticity fields at $t=6 \tau$ for three angles of incidence, $\alpha=0^{\circ}, 30^{\circ}, 45^{\circ}$.
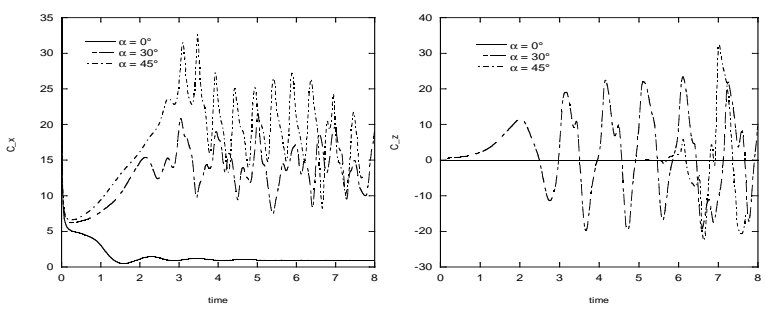

Figure 6: Flow past tube bundles with square cross section at $R e=200$ for $\alpha=0^{\circ}, 30^{\circ}, 45^{\circ}$. Time evolution of drag (left) and lift (right) coefficients.

\section{CONCLUSIONS AND PERSPECTIVES}

We presented a numerical scheme for computing the time evolution of two-dimensional flows in complex geometries. The utilisation of a volume penalisation method enables us to take into account complex geometries using a mask function without modifying 
the numerical scheme.

We applied the numerical method to different industrially relevant devices, such as heat-exchangers and chemical reactors. We studied the transient flow behaviour in tube bundles with circular and square cross sections at Reynolds number 200 for different angles of attack. The two-dimensional approximation of the simulation can be justified in this case due to the dense packing of the tubes. The numerical scheme is able to resolve the thin shear layers formed at the tube's wall, which become unstable and lead to the formation of vortices. Depending on the parameters, we observed strong oscillations of the lift and drag forces exerted by the flow on the tubes. These flow induced oscillations may dammage the tubes (cf. (Axisa et al., 1990)), especially when the vortex shedding frequency is close to the tubes' resonance frequency.

The adaptive wavelet method presented in this paper allows automatic grid generation and refinement around the obstacles and also in shear layers which develop during the flow evolution. Therewith the number of required grid-points in the simulations is significantly reduced. We conjecture that the compression rate thus obtained increases with the Reynolds number.

In future work we will extend the penalisation scheme to compute fluid-structure interaction in three-dimensional flows and perform computations at high Reynolds numbers using the Coherent Vortex Simulation approach, proposed by Farge and Schnei$\operatorname{der}(2001)$.

Acknowledgements: We thank Nicholas Kevlahan for fruitful discussion and for help in developing the pseudo-spectral code. We also acknowledge financial support from the European program IHP 'Breaking complexity' (contract HPRN-CT 2002-00286) and from the fusion program of CEA-Euratom, Cadarache.

\section{REFERENCES}

F. Axisa, J. Antunes and B. Villard. Random excitation of heat exchangers tubes by cross flows. J. Fluids Struct. 4:321-341, 1990.

P. Angot, C.-H. Bruneau and P. Fabrie. A penalisation method to take into account obstacles in viscous flows. Num. Math.,81, 497-520, 1999.

E. Arquis and J.P. Caltagirone. Sur les conditions hydrodynamiques au voisinage d'une interface milieu fluide - milieux poreux: application à la convection naturelle. C. R. Acad. Sci. Paris II, 299, 1-4, 1984.
S.B. Beale and D.B. Spalding. A numerical study of unsteady flow in in-line and staggered tube banks. $J$. Fluids Structures, 13, 723-754, 1999.

C. Canuto, M.Y. Hussaini, A. Quaternioni and T. A. Zang. Spectral Methods in Fluid Dynamics, Springer-Verlag, Berlin Heidelberg, 1988.

M. Farge. Wavelet transforms and their applications to turbulence. Ann. Rev. of Fluid Mech. 24, 395-457, 1992.

M. Farge and K. Schneider. Coherent Vortex Simulation (CVS), a semi-deterministic turbulence model using wavelets. Flow, Turbulence and Combustion, 66(4), 393-426, 2001.

J. Fröhlich and K. Schneider. An adaptive waveletvaguelette algorithm for the solution of PDEs. $J$. Comput. Phys., 130, 174-190, 1997.

M. Forestier, R. Pasquetti and R. Peyret. Calculations of 3D wakes in stratified fluids. ECCOMAS, 2000.

N. Kevlahan and J.-M. Ghidaglia. Computation of turbulent flow past an array of cylinders using a spectral method with Brinkman penalization. Eur. J. Mech./B, 20, 333-350, 2001.

A. Khalak and C. Williamson. Motions, forces and dynamics of hydroelastic structure with very low mass damping. J. Fluids Struct. 13:813-851, 1999.

K. Khadra, S. Parneix, P. Angot and J.-P. Caltagirone. Fictious domain approach for numerical modelling of Navier-Stokes equations. Int. J. Num. Meth. Fluids, 34, 651-684, 2000.

K. Schneider and M. Farge. Adaptive wavelet simulation of a flow around an impulsively started cylinder using penalisation. Appl. Comput. Harm. Anal., 12, 374-380, 2002.

K. Schneider. Numerical simulation of the transient flow behaviour in chemical reactors using a penalisation method. Comput. Fluids, accepted 2004. 